\title{
Cuoricino and CUORE detectors: developing big arrays of large mass bolometers for rare events physics
}

P. Gorla , R. Ardito , C. Arnaboldi , D. R. Artusa , F. T. Avignone III , M. Balata ,

I. Bandac , M. Barucci , J. Beeman , C. Brofferio , C. Bucci , S. Capelli , F. Capozzi ,

L. Carbone , S. Cebrian , O. Cremonesi , R. J. Creswick , M. Dolinski , A. de Waard ,

H. A. Farach ,F. Ferroni , E. Fiorini , G. Frossati , C. Gargiulo , A. Giuliani , E. Guardincerri ,

T. Gutierrez , E. E. Haller ，I. G. Irastorza ，E. Longo ，G. Maier ，R. Maruyama ,

R. J. McDonald , S. Morganti , A. Morales , S. Nisi , E. B. Norman , A. Nucciotti , E. Olivieri ,

P. Ottonello , M. Pallavicini , V. Palmier , E. Pasca , M. Pavan , M. Pedretti , G. Pessina ,

S. Pirro , E. Previtali , B. Quiter L. Risegari , C. Rosenfeld , S. Sangiorgio , M. Sisti ,

A. R. Smith , . Toffanin , L. Torres , G. Ventura , and N. Xu

In the last 10 years bolometers have become extremely powerful detectors in the search for rare events, due to their very good resolution. Cuoricino, a 62 bolometer array, in the first months of activity reached a resolution (average in all detectors) in the energy region of interest $(2528 \mathrm{keV})$ of $7 \mathrm{keV}$ and a limit on the $t_{1 / 2}$ of the process of $7.5 \cdot 10^{23} \mathrm{y}$. Cuoricino's 62 detectors constitute the biggest number of macrobolometers (790 g each) ever cooled and demonstrate the feasibility of large arrays of bolometers. Following the indication of Cuoricino in CUORE R\&D a new detector has been developed trying to improve the reproducibility of the detector's performance, to increase the single detector mass (to reduce the number of channels) and to simplify the experiment assembly. The latest results are presented. 


\section{Introduction}

Double beta decay (DBD) consists of a rare transition from the nucleus $(A, Z)$ to its isobar $(\mathrm{A}, \mathrm{Z}+2)$ with the emission of two electrons. It can be observed when the transition from $(\mathrm{A}, \mathrm{Z})$ to $(A, Z+1)$ is energetically forbidden or at least strongly suppressed by a large change of angular momentum. Two-neutrino double beta decay, where two anti-neutrinos are emitted together with the two electrons, conserves the lepton number, is predicted by the standard weak interaction theory and has been observed for several nuclei (with typical $t_{1 / 2} \sim 10^{19}-10^{21} \mathrm{y}$ ). If lepton number is violated, the two electrons could be emitted without any other particle. This process, called neutrinoless double beta decay $(0 \nu$-DBD), would have obvious implications for extended particle models and beyond Standard Model physics.

\section{Experimental approach}

Cuoricino (and CUORE) are experiments for measuring $0 \nu$-DBD of ${ }^{130} \mathrm{Te}$, using bolometric detectors with the so called calorimetric approach. This expression usually refers to experiments in which the source of the searched event is the detector itself (or at least part of it). In Cuoricino the absorber crystal of the bolometers is a crystal of $\mathrm{TeO}_{2}$ which obviously contains the isotope of interest (isotopic abundance $33.8 \%$ ). This means that, when a ${ }^{130} \mathrm{Te}$ atom decays inside the crystal, the two electron release all of their energy inside the absorber so that an energy line is expected at the transition energy Q of the process $(2528 \mathrm{keV}$ in this case) for $0 \nu$-DBD. In the 2 neutrino case, where part of the energy escapes with neutrinos, a continuous spectrum between 0 and $\mathrm{Q}$ with a peak at about $1 / 3$ of $\mathrm{Q}$ will appear (see Fig. 1).

\subsection{Bolometers for Cuoricino and CUORE}

Bolometric detectors in Cuoricino are realized using a $\mathrm{TeO}_{2}$ cubic crystal of $5 \mathrm{~cm}$ in size, held with small PTFE supports that provide weak thermal coupling to the heat reservoir, a copper structure that is connected to the coldest part of

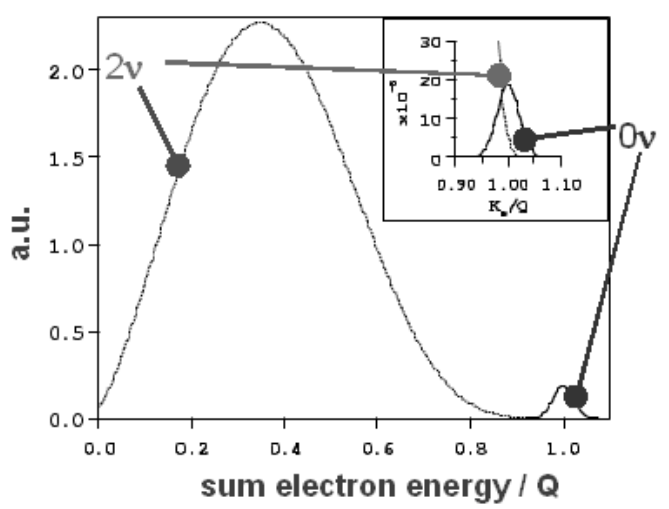

Figure 1. Energy spectrum for $0 \nu$ - and $2 \nu$-DBD. To resolve the $0 \nu$ peak is necessary a resolution at least better than $2 \%$.

a dilution refrigerator. When a radiation releases an energy $\mathrm{E}$ inside the absorber the temperature increases proportionally $(\Delta \mathrm{T}=\mathrm{E} / \mathrm{C}$ where $\mathrm{C}$ is the heat capacity of the crystal) and is measured by a Neutron Transmutation Doped (NTD) germanium thermistor.

\subsection{Sensitivity}

The sensitivity of such a detector for $0 \nu$-DBD can be easily estimated as the lifetime corresponding to the minimum number of detectable events above background at a given C.L.. This gives:

$F \propto \sqrt{\frac{M \cdot t}{b \cdot \Delta E}}$

where $\mathrm{M}$ is the total active mass of the detector, $\mathrm{t}$ the measurement time, $\mathrm{b}$ the the background (in counts $/ \mathrm{keV} / \mathrm{kg} / \mathrm{y}$ ), and $\Delta \mathrm{E}$ the energy resolution in the region of interest. It is obvious that to improve the sensitivity it is necessary to focus $\mathrm{R} \& \mathrm{D}$ activity on three parameters: the mass, the background, and the resolution.

\subsubsection{Mass of the detector}

Increasing the mass of the detector (i.e. the number of atoms of the candidate isotope) is the 
easiest way of increasing sensitivity. This could be done in two ways: increase the single detector mass or increase the number of detectors.

The first task improved in the last 15 years by more than an order of magnitude (see Table below).

\begin{tabular}{lll}
\hline year & mass $(\mathrm{g})$ & dimensions $\left(\mathrm{cm}^{3}\right)$ \\
\hline 1991 & 73 & - \\
1993 & 340 & $3 \times 3 \times 6$ \\
2000 & 790 & $5 \times 5 \times 5$ \\
2004 & 1300 & $6 \times 6 \times 6$ \\
\hline
\end{tabular}

The only limitation of this increase is the fact that the heat capacity grows linearly with mass: this means that the temperature variation, for the same released energy (and pulse height), decreases linearly with growing mass. Nevertheless this is not a real problem, since at present noise is dominated by thermal effects, like baseline oscillation due to energy dissipation from mechanical vibrations in the apparatus. So the noise and the pulse height are both reduced by the same factor with the increasing $\mathrm{C}$, and the $\mathrm{S} / \mathrm{N}$ is maintained, without any loss of resolution.

The actual limitation is given by growing such big crystals maintaining quality and mechanical properties. The best compromise is the choice of $5 \times 5 \times 5 \mathrm{~cm}^{3}$ crystals of $790 \mathrm{~g}$ in mass.

The number of detectors has also increased from a 4 detector array in 1993 up to the present 62 detectors of Cuoricino with a total mass of $\mathrm{TeO}_{2}$ of $40.9 \mathrm{~kg}$. The final step of this evolution will be the recently approved CUORE project, which will be an array of 1000 detectors for a total mass of $790 \mathrm{~kg}$.

\subsubsection{Background}

Background reduction is a primary task for all experiments in low energy physics (especially for those which have no pulse shape discrimination). The biggest source of background for $\mathrm{TeO}_{2}$ detectors come from escaping radiation (alphas) which are generated nearby the surface and that leave only part of their energy in the absorber, generating a continuum of events at every energy. CUORE detector is improved by using an anticoincidence technique to solve this problem. The basic idea is to increase as much as possible

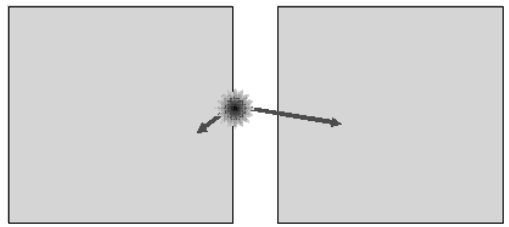

Figure 2. Anticoincidence method sketch: a nuclear disintegration happens on the surface of one detector releasing energy into it; the escaped radiation is detected by a second detector.

the amount of active surface (i.e. absorber crystals surface) facing active surface, so that multi events, in time coincidence, can be reconstructed (sum energy) or rejected (see Fig.2).

This technique is tested in Cuoricino in two spacial layers of the tower that consist in a 9 detectors modules in which the central crystal of each is completely surrounded of active material.

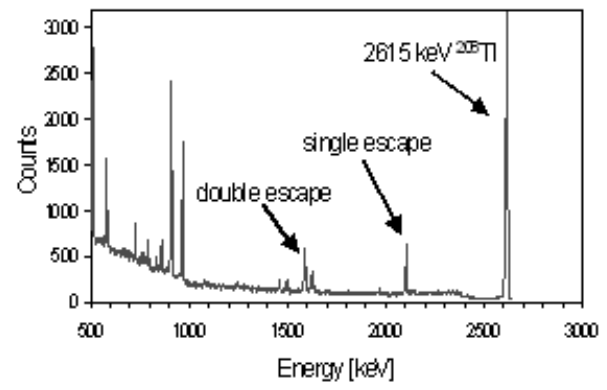

Figure 3. Sum spectrum of all the $5 \times 5 \times 5 \mathrm{~cm}^{3}$ crystal obtained during a ${ }^{232} \mathrm{Th}$ calibration.

\subsubsection{Energy resolution}

The Cuoricino bolometers where optimized for energy resolution through years (temperature stabilization, vibration filtering, etc.). In 2000 the 
best resolution ever was obtained with alpha particles [1]. Cuoricino demonstrate the possibility of extending good performance to a big number of detectors. In fact the average pulse height is about $440 \mathrm{~V} / \mathrm{MeV}$ for the $5 \times 5 \times 5 \mathrm{~cm}^{3}$ crystals and $340 \mathrm{~V} / \mathrm{MeV}$ for the $3 \times 3 \times 6 \mathrm{~cm}^{3}$ crystals. The array has been calibrated exposing it to a ${ }^{232} \mathrm{Th}$ radioactive source; the FWHM energy resolution at $2615 \mathrm{keV}$ ranges between 4 and $20 \mathrm{keV}$ with an average of 7 and $9 \mathrm{keV}$ respectively for the 790 $\mathrm{g}$ and the $330 \mathrm{~g}$ detectors. The good uniformity of the detectors is obvious, looking at the sum spectrum of the $5 \times 5 \times 5 \mathrm{~cm}^{3}$ crystals in Fig. 3 .

\section{Cuoricino detector}

The Cuoricino experiment, operating in Hall A of the Gran Sasso Underground Laboratory, is a natural extension of the Milano Double Beta Decay experiment, completed in 2001 [2]. Cuoricino consists of a tower with 13 layers (see Fig. 4) of which 11 layers are realized with $4 \mathrm{TeO}_{2}$ crystals of $5 \times 5 \times 5 \mathrm{~cm}^{3}(790 \mathrm{~g})$, while 2 layers are composed of $9 \mathrm{TeO}_{2}$ crystals of $3 \times 3 \times 6 \mathrm{~cm}^{3}(330 \mathrm{~g})$ for a total mass of about $40.9 \mathrm{~kg}$. A resistor of $50-100 \mathrm{k}$, realized with a heavily doped meander on a $1 \mathrm{~mm} 3$ silicon chip is also attached to each absorber and acts as an heater to calibrate and stabilize the gain of the bolometer [3].

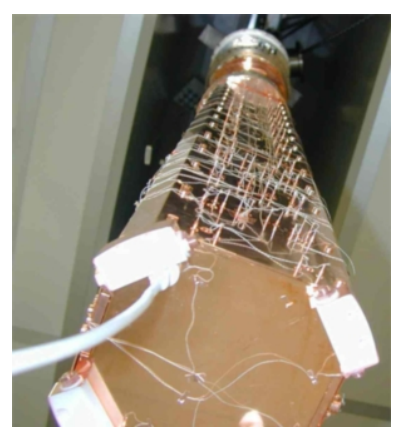

Figure 4. Wiew of Cuoricino (from bottom) during installation.

\subsection{Results on $0 \nu$-DBD}

The good performances of Cuoricino allowed, in slightly more than a month of data collection $(5.4 \mathrm{~kg} \cdot \mathrm{y})$, to improve the previous results. No evidence was found for neutrinoless double beta decay of ${ }^{130} \mathrm{Te}$ with a $90 \%$ C.L. lower limit of $7.5 \cdot 10^{23}$ years; this corresponds to an upper limit on the effective neutrino mass $\langle\mathrm{m}\rangle$ ranging from 0.31 to $1.63 \mathrm{eV}$, depending on the various theoretical calculations of the nuclear matrix elements. Beyond the physics results, Cuoricino is also a test of feasibility of a bigger experiment [4] called CUORE (Cryogenic Underground Observatory for Rare Events) that will be composed by $1000 \mathrm{TeO} 2$ detectors for a total mass of about 0.8 ton.

\section{Prospective and conclusions}

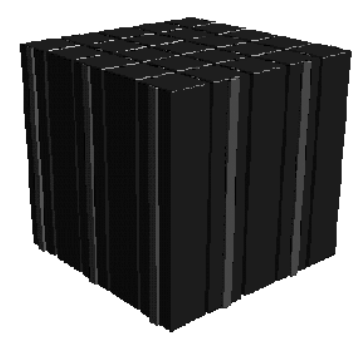

Figure 5. Sketch of Cuore, a cubic structure of 25 Cuoricino-like towers.

Cuoricino demonstrates the feasibility of a large mass bolometric experiment. CUORE will be the first second generation experiment for $0 \nu$ DBD. The structure of this experiment will improve anticoincidence for most of the crystals with the big cubic structure reducing as much as possible non active materials. Big improvment is expected in background reduction $(1 \mathrm{c} / \mathrm{keV} / \mathrm{ton} / \mathrm{y})$ and in sensitivity $(\langle\mathrm{m}\rangle \sim 15-40 \mathrm{meV})$. 


\section{REFERENCES}

1. M. Vanzini et al., Nucl. Instrum. Meth. A 461, 293 (2001).

2. C. Arnaboldi et al., Phys. Lett. B 557, 167 (2003)

3. A. Alessandrello et al., Nucl. Instrum. Meth. A 412, 454 (1998).

4. C. Arnaboldi et al. [CUORE Collaboration], Astropart. Phys. 20, 91 (2003) 